Article

\title{
Wenner Soundings for Apparent Resistivity Measurements at Small Depths Using a Set of Unequal Bare Electrodes: Selected Case Studies
}

\author{
Eduardo Faleiro *(D), Gabriel Asensio, Gregorio Denche, Daniel Garcia and Jorge Moreno \\ Departamento de Ingeniería Eléctrica Electrónica, Automática y Física Aplicada, Escuela Técnica Superior de \\ Ingeniería y Diseño Industrial, Universidad Politécnica de Madrid, 28012 Madrid, Spain; \\ gabriel.asensio@upm.es (G.A.); gdenche@ree.es (G.D.); daniel.gpuertas@upm.es (D.G.); \\ jorge.moreno@upm.es (J.M.) \\ * Correspondence: eduardo.faleiro@upm.es; Tel.: +34-91-336-7686
}

Received: 17 January 2019; Accepted: 18 February 2019; Published: 21 February 2019

check for updates

\begin{abstract}
The possibility of using a set of unequal electrodes, within limits, in a Wenner arrangement for the measurement of apparent resistivity at small depths is explored in this paper. A procedure in which only a simple preliminary calibration is necessary to obtain the best measurements of the apparent resistivity is proposed. On the basis of some case studies, a comparison with the usual procedures to obtain the apparent resistivity from resistance measurements is carried out. The results showed that when an unequal set of electrodes was used, the procedure proposed here was the only one that guaranteed the best apparent resistivity values for any value of electrode separation in the Wenner arrangement, especially for those associated with small depths.
\end{abstract}

Keywords: vertical electrical sounding; grounding resistance; multilayer soil model; apparent resistivity measurements

\section{Introduction}

One of the most frequently used methods to determine the electrical resistivity of the ground versus the depth is vertical electrical sounding (VES) [1]. By means of electrode arrangements—such as Wenner, Schumberger, dipole-dipole, and aligned quadrupolar arrays, among others-it is possible to explore the variation of the electrical properties of soil with depth by simply varying the distance between the electrodes in the array [2]. In particular, the resistivity at shallow depths of the ground is of special interest in archeology, geophysics, and electrical engineering. This resistivity is generally not well measured using these types of arrangements, mainly because the electrodes are usually treated as point electrodes, while their finite size is ignored. Some attempts to improve this situation use a non-aligned quadrupole array [3] or other more sophisticated techniques, which are significantly different to those that are studied in this paper [4]. Although this paper focuses on the aligned Wenner arrangement, the results can be extended to other arrangements of interest, such as the Schumberger arrangement.

In the Wenner arrangement, four equal aligned electrodes are arranged separated from each other by the same distance, $a$. Two of the electrodes are activated as the injection point and the return point of a fixed current, I, while the voltage difference, $\Delta V(a)$, between the other two electrodes, which has been generated by the artificially generated electric field, is measured [5]. The four electrodes are usually arranged with the active electrodes on the outside, although other configurations are possible. The survey is carried out by increasing the separation distance, $a$, but keeping the distances between the electrodes in the array equal. Other Wenner arrangements either locate the electrodes in a 
semi-circle, in order to avoid obstacles in the ground, or arrange them unaligned to take full advantage of the limited soil surface for measurement purposes [6]. In any case, it is usually necessary to have a set of equal electrodes and a device to inject current through the active electrodes while at the same time being able to measure either $\Delta V(a)$ or the resistance between the measuring electrodes $[7,8]$. Regarding the electrodes, well-conductive metal rods that are stuck in the ground, of length $\mathrm{L}=0.3 \mathrm{~m}$, are used. The VES is carried out by separating the rods from a recommended initial value of $a>10 \cdot L$ to several tens of meters. For each value of the electrode separation $a, \Delta V(a)$ is measured and the apparent resistivity is obtained from $\rho_{a p p}=F(a) \cdot(\Delta V(a) / I)$, where $F(a)$ is a function that we will here call the geometric function, which depends on the geometric shape of the electrodes used in the VES. $\Delta V(a) / I$ is the apparent electrical resistance of the conductive soil between the points where the potential difference is measured. The distance between the electrodes is usually set to $F(a)=2 \pi a$, which is equivalent to considering the electrodes as current points at ground level. If depths smaller than those associated with the previous values of the separation $a$ are to be explored, it is suggested to use $F(a)$ for the Equation [9]:

$$
F(a)=\frac{4 \pi a}{1+\frac{2 a}{\sqrt{a^{2}+4 L^{2}}}-\frac{a}{\sqrt{a^{2}+L^{2}}}}
$$

which basically considers the electrodes as current point at depth $\mathrm{L}$ and reduces to $F(a)=2 \pi a$ when $L \rightarrow 0$. However, in a recent paper [10], the authors showed that if the bare rods described above are used as electrodes, then the two previous expressions of $F(a)$ for the calculation of the apparent resistivity do not give the correct values of this magnitude for electrode separations of $a<10 \mathrm{~L}$. This issue is very significant because it involves depths that are important, either from the technical or scientific point of view. The incomplete knowledge of the electrical structure at small depths can lead, for example, to significant errors in the calculation of the grounding resistance of the protection systems. Consider, for example, a synthetic three-layer model with parameters $\rho_{1}=100 \Omega \mathrm{m}, \rho_{2}=20 \Omega \mathrm{m}$, $\rho_{3}=50 \Omega \mathrm{m}, h_{1}=1 \mathrm{~m}$, and $h_{2}=3 \mathrm{~m}$. If a Wenner VES is made using equal bare rods as electrodes $\mathrm{L}=0.3 \mathrm{~m}$ long and $\mathrm{r}=0.005 \mathrm{~m}$ radius with the distance $a$ between electrodes ranging from $0.1 \mathrm{~m}$ to $13 \mathrm{~m}$, one can obtain either the curve in Figure 1 that is labeled 'points' (corresponding to use as a geometrical function $F(a)=2 \pi a$, which is associated with point electrodes as probes at ground level) or the curve labeled IEEE, in which Equation (1) (which is associated with point electrodes buried at a depth $\mathrm{L}$ ) is used for $F(a)$. The curve labeled 'theoretical' corresponds to the apparent resistivity associated with the proposed synthetic model. This preliminary example emphasizes the importance of using the appropriate $F(a)$ function if one wants to reproduce the apparent resistivity correctly, especially for small values of the electrode separation $a$, as is shown in the subfigure in Figure 1.

In view of Figure 1, the need to perform some type of electrode calibration is claimed in order to find an adequate $F(a)$ function. The calibration is a really simple operation that provides the key to convert the values of the potential, $\Delta V(a)$, into values of the apparent resistivity of the ground, $\rho_{a p p}(a)$, for any value of the separation between electrodes $a$. As is demonstrated in this paper, the calibration is not limited to a set of equal electrodes. Almost any type of electrode can be part of a Wenner arrangement whenever a distance between electrodes $a$ can be defined so that it can be varied in order to carry out the electrical survey of the ground. 


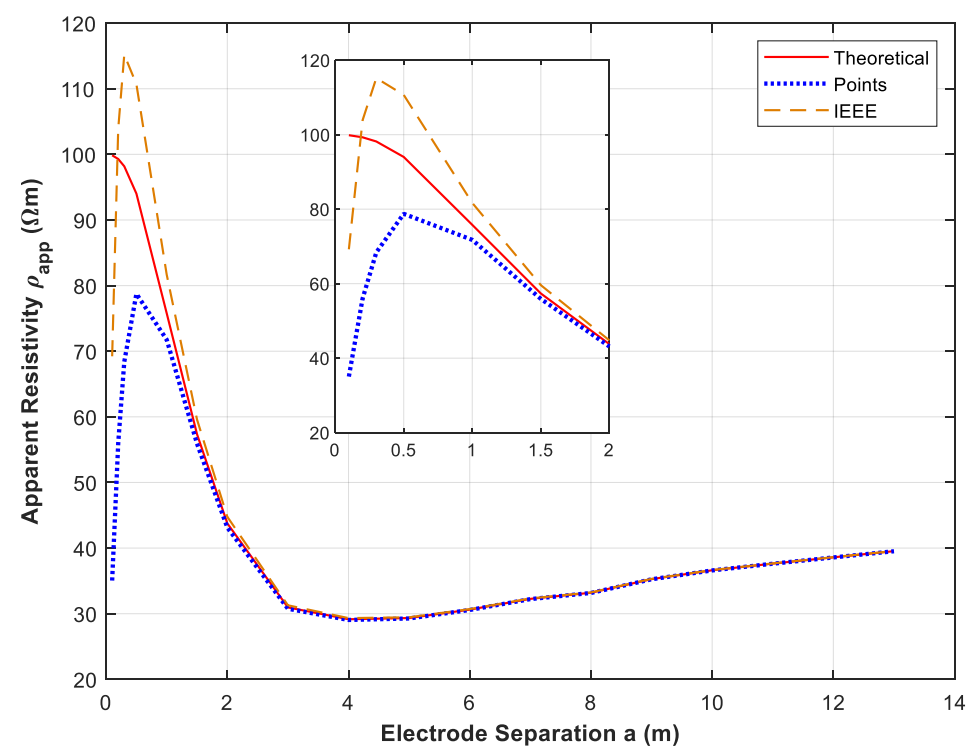

Figure 1. Apparent resistivity curves for the synthetic three-layer soil defined in the text. The continuous line curve, labeled 'theoretical', corresponds to the real value of the apparent resistivity for any value of the electrode separation.

As a motivation for the present study, let us suppose that a complete set of electrodes for the Wenner sounding is not available. In this paper it is shown that almost any elongated metallic object can serve as a probe, together with the rest of the available electrodes. Another situation, which can take place in this study, arises when, for various reasons, it is not possible to bury all the electrodes of the Wenner arrangement at the same depth while subsurface exploration at small depths is necessary. In any case, whenever it is necessary to obtain a real measurement of the resistivity at shallow depth by using electrodes of varying shape and size that are introduced into the ground at a not negligible depth, it is necessary to make a correction in the measurements. This is related to the calibration proposed in this work.

In summary, in the present paper it is shown that it is possible to use electrodes of varied shapes and sizes for Wenner arrangements, as long as the geometrical function, $F(a)$, is adequately defined either analytically or numerically. With a selected set of electrodes, the apparent resistivity, obtained by applying the commonly used expressions for $F(a)$, is compared with that obtained from the calibration. For this purpose, the paper is organized as follows: After the introduction to the problem in section, the theoretical foundation is sketched in Section 2. In Section 3, some case studies and subsequent analyses are presented. Finally, the conclusions of this work are summarized in Section 4.

\section{Theoretical Background}

The theoretical foundations on which this work is based have been well established for decades, except for some necessary improvements related to the peculiarities of how some problems are treated [10]. Starting from a current point in a multilayered soil, the Poisson equation for the electric potential must be solved within the layer containing the source and the Laplace equation is used for the other layers, in addition to imposing the boundary conditions at each interface. It is usual to use the separation of variables method for cylindrical coordinates to obtain a general solution, to which the boundary conditions will be applied [11].

To deal with real electrodes, the thin wire approach is used, which allows for the application of the moments method [12] for the numerical resolution of the problem. In a first stage, the distribution of current filtered to the ground by the electrode is calculated by imposing a constant value of the potential on the electrode. Subsequently, the potential and the electric field, at any point on the ground, 
can be calculated without difficulty. Some details concerning the shape, thickness, or size of the electrodes, should be treated carefully within the general calculation scheme.

Based on the Maxwell equations and using the moments method, the authors of this paper wrote a MatLab code (which contains the appropriate modifications to deal with almost any type of electrode, always within the thin wire approach [10]) that performed all the necessary calculations for multilayered soils.

\section{Application to Selected Case Studies}

As mentioned in the introduction, once the set of electrodes has been selected to carry out the survey, the first step is to calibrate them. The purpose of the calibration operation is to find the appropriate geometrical function to implement the conversion between either the potential or resistance of the measuring electrodes, supplied by the measuring device, to values of the apparent resistivity. The basis of the calibration method is simple. The electrodes must comply with the thin wire approach and the common distance between electrodes, $a$, to be varied must be clearly identified in the arrangement. The selected electrodes are buried in a soil of homogeneous resistivity, $\rho$, and a simulated VES is performed to find the values of the potential difference, $\Delta V(a)$, for a complete range of values of the electrode separation $a$. The geometrical function sought is obtained numerically by the expression $F(a)=\rho I / \Delta V(a)$.

Figure 2 shows the system of electrodes used in the simulation. The geometrical characteristics, length, $L$, and radius, $r$, of the electrodes, as well as the number of segments $M$ into which each electrode was divided, are shown in Table 1.

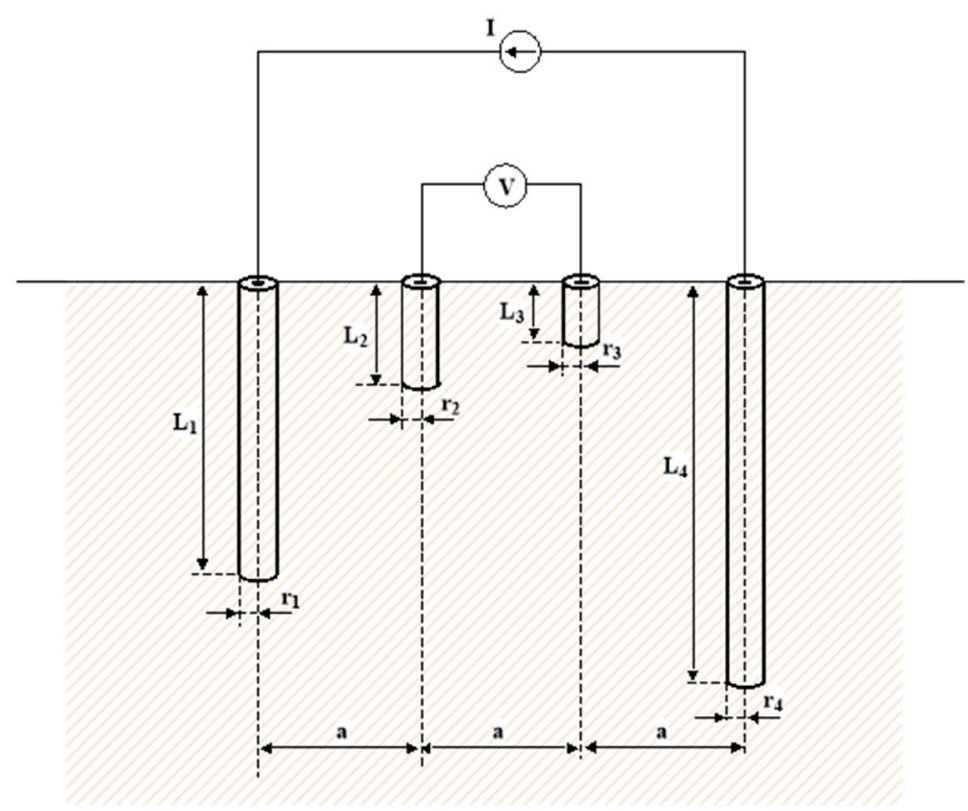

Figure 2. Four-pin Wenner arrangement to simulate the vertical electrical sounding (VES). For the determination of the geometrical function $F(a)$, the Wenner probe is placed in a homogeneous semi-conductive medium.

Table 1. Geometrical features (GF), length and radius of the Wenner arrangement electrodes. The number of segments of each electrode is $\mathrm{M}$.

\begin{tabular}{ccccc}
\hline \multicolumn{5}{c}{ Electrode } \\
\hline $\mathbf{G F}$ & $\mathbf{1}$ & $\mathbf{2}$ & $\mathbf{3}$ & $\mathbf{4}$ \\
$\boldsymbol{L}(\mathbf{m})$ & 0.2 & 0.02 & 0.01 & 0.9 \\
$\boldsymbol{r}(\mathbf{m})$ & 0.007 & 0.01 & 0.01 & 0.005 \\
$\mathbf{M}$ & 20 & 2 & 1 & 30 \\
\hline
\end{tabular}


Figure 3 shows the values of $F(a)$ for 150 values of the electrode separation, $a$, ranging from 0.02 to $15 \mathrm{~m}$. The function is almost rectilinear from $a>4 \mathrm{~m}$. In the subfigure, the values of $F$ for $a<1.5 \mathrm{~m}$ are represented. That region corresponds to the critical zone for $F(a)$, where clear differences between the various methods of calculating the apparent resistivity were found. The result did not change significantly if the segmentation was refined.

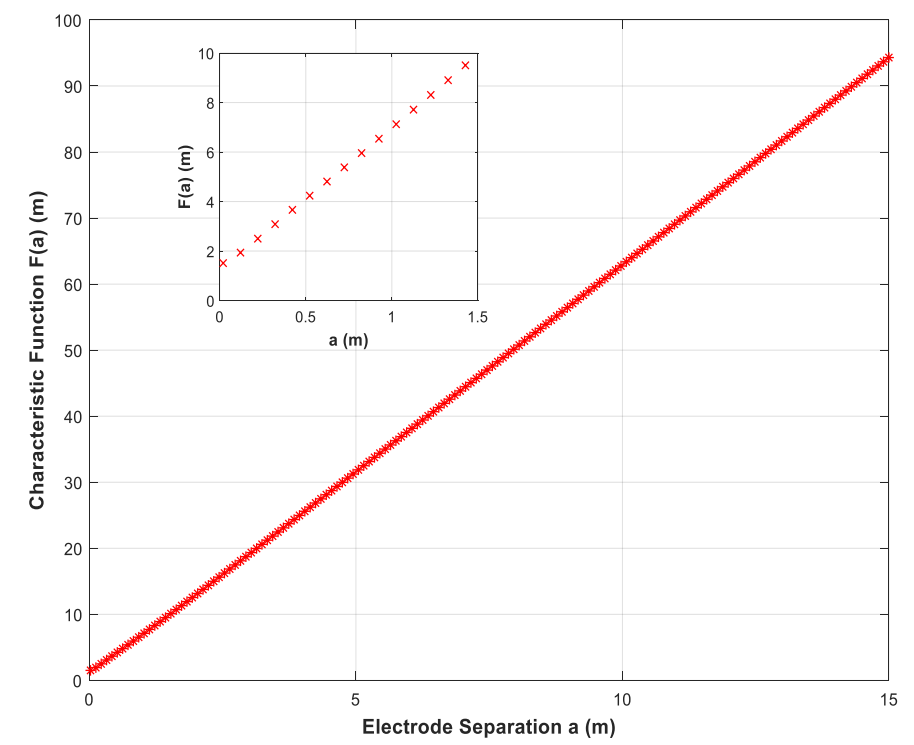

Figure 3. Four-pin Wenner geometrical function $F(a)$ vs. $a$ for the considered set of electrodes.

Once the Wenner probe with the real electrodes is calibrated, the VES sounding on multi-layered soils can be performed in order to find either the potential, $\Delta V(a)$, or the resistance, $R(a)=\Delta V(a) / I$, between the measurement electrodes. Finally, the apparent resistivity is obtained from

$$
\rho_{\text {app }}(a)=R(a) \cdot F(a) .
$$

Note that the calibration is not restricted to straight rods and can be extended to any type of electrode, provided that it can be modeled as a structure of thin wires and a parameter equivalent to $a$ can be defined.

In the first two case studies, presented below, a real sounding, performed with identical electrodes to obtain a multilayer model of the soil, was the starting point. The set of identical electrodes were bare rods buried at ground level, with a $5 \mathrm{~mm}$ radius and length of $0.3 \mathrm{~m}$, which were calibrated to obtain the correct value of the apparent resistivity at any value of separation between electrodes. Next, a simulated sounding was performed on the previously modeled ground, using the set of electrodes seen in Figure 2, which shows that the obtained apparent resistivity curves fit well with the data. The third case study was completely synthetic, with the purpose of showing that almost any electrode is valid as a probe in a Wenner arrangement.

\subsection{Case Study 1}

Using a C.A. 6472 Chauvin-Arnoux resistivity tester and equal vertical bare rods (of $L=0.3 \mathrm{~m}$ and $r=0.005$ ) as electrodes, a VES involving four values for the electrode separation, $a=2,5,10$, and $15 \mathrm{~m}$, was performed at a site located in Madrid (Spain). Although there were few available measures, a two-layered model, with parameters $\rho_{1}=23.41 \Omega \mathrm{m}, \rho_{2}=12.05 \Omega \mathrm{m}$ and $h=3.11 \mathrm{~m}$, was proposed by the authors [10].

Next, a simulated VES sounding, with the set of electrodes as shown in Figure 2, was performed on the proposed two-layered soil. Figure 4 shows the apparent resistivity, $\rho_{a p p}(a)$, for the different models of the geometrical function $F(a)$ namely, a model corresponding to point electrodes at ground level 
(labeled 'points' in Figure 4); a model proposed in Equation (1) and associated with point electrodes all buried at a depth $h_{1}$ as the average value of all the electrode lengths (labeled IEEE1); a model that is the same as the previous model but considering a burial depth $h_{2}$ as the average length of only the longest electrodes (labeled IEEE2 in Figure 4); and the model proposed in this paper from Equation (2) by taking the geometrical function, $F(a)$, from Figure 3 and associating it with the actual set of electrodes used in the simulated sounding (labeled 'real' in Figure 4). It can be clearly seen that from $a>4 \mathrm{~m}$ all resistivity curves are nearly coincident, whilst for lower values a very different behavior is apparent. Only the curve labeled 'real', where we used an electrode calibration resulting from the geometrical function, most closely resembles the theoretical resistivity curve associated with a two-layer model, as was proposed, for all values of separation $a$ between electrodes.

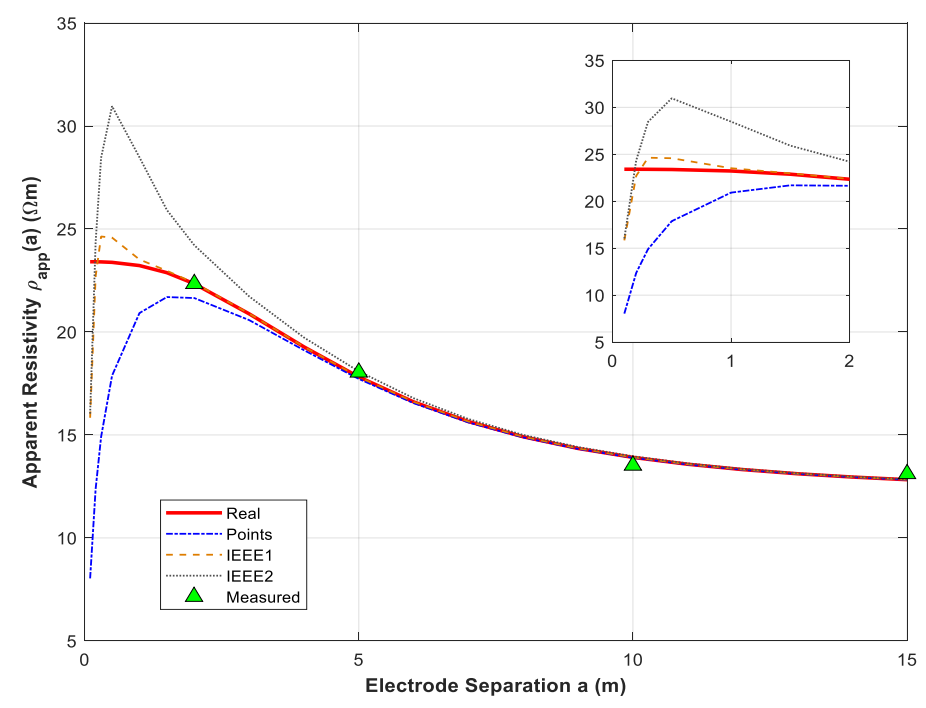

Figure 4. Curves of apparent resistivity vs. the separation $a$ between the electrodes, for the measured site in Madrid (Spain), according to a two-layer soil model. Beside the actual measured data (green triangles), those simulated using the geometrical function $F(a)$, proposed in the text, are shown.

Related to the IEEE model, the IEEE1 curve associated with the average length $h_{1}$, was the closest to the true apparent resistivity, at least for $a>1 \mathrm{~m}$, whereas the IEEE2 curve was only close for $a>5 \mathrm{~m}$, which indicates that the value of parameter $L$ in the model of Equation (1) is critical. Even for equal electrodes, the length $L$ in Equation (1) must be less than the length of the largest electrode. It is not easy to determine $L$ accurately so that the apparent resistivity curve approaches the curve labeled 'real'. Similar results were obtained if the active electrodes were smaller than the measuring electrodes. Additionally, similar results were obtained by assuming that each individual electrode had a variable radius, as long as the thin wire approximation in the calculations remained valid. Although it is not one of the objectives of this paper, Figure 4 indicates that if the standard geometric factor is used, which corresponds to considering the probes as point electrodes, the soil model would correspond to a three-layer soil, with the upper layer having a very low resistivity and thickness. Applying our inversion algorithm $[13,14]$ results in $\rho_{1}=6.89 \Omega \mathrm{m}, \rho_{2}=27.31 \Omega \mathrm{m}, \rho_{3}=12.59 \Omega \mathrm{m}, h_{1}=0.13 \mathrm{~m}$ and $h_{2}=1.3 \mathrm{~m}$.

\subsection{Case Study 2}

The following presents a second application case using a three-layered model. Measurements made on a site located in Vitoria (Spain) are shown in Table 2. For this purpose, a set of equal electrodes, $0.3 \mathrm{~m}$ in length and with a $0.005 \mathrm{~m}$ radius, were used in field measurements. Table 2 shows the measured values of the resistance and the geometrical function for the considered set of equal electrodes. 
Table 2. Measured resistance vs. the electrode separation, $a$, for Vitoria (Spain). The values for the geometrical function associated with the set of equal bare rods, $0.3 \mathrm{~m}$ in length and with a $0.005 \mathrm{~m}$ radius, are $F(a)$.

\begin{tabular}{ccc}
\hline $\mathbf{a}(\mathbf{m})$ & $\mathbf{R}(\mathbf{a})(\boldsymbol{\Omega})$ & $\mathbf{F}(\mathbf{a})(\mathbf{m})$ \\
\hline 0.5 & 13.64 & 3.75 \\
1 & 6.87 & 6.64 \\
1.5 & 4.69 & 9.67 \\
2 & 3.51 & 12.75 \\
3 & 2.88 & 18.97 \\
4 & 2.29 & 25.23 \\
6 & 1.76 & 37.76 \\
8 & 1.40 & 50.31 \\
10 & 1.25 & 62.87 \\
12 & 1.07 & 75.43 \\
\hline
\end{tabular}

After calibrating the electrodes, the apparent resistivity was calculated and a three-layer model with parameters $\rho_{1}=53.62 \Omega \mathrm{m}, \rho_{2}=33.79 \Omega \mathrm{m}, \rho_{3}=89.82 \Omega \mathrm{m}, h_{1}=0.61 \mathrm{~m}$, and $h_{2}=1.25 \mathrm{~m}$ was estimated $[13,14]$. Next, a set of non-equal electrodes similar to that of Table 1 but with $L_{4}=0.5 \mathrm{~m}$ was used to simulate a VES in a three-layered soil with those parameters. The main reason for shortening electrode 4 was to not involve the second layer of the ground when performing the VES. Table 3 shows the values of the simulated resistance and those of the geometrical function after calibration for the same values of $a$ as in Table 2, although in practice some further values were considered.

Table 3. Simulated resistance vs. the electrode separation, $a$, for Vitoria (Spain). The values for the new geometrical function are $F(a)$.

\begin{tabular}{ccc}
\hline $\mathbf{a}(\mathbf{m})$ & $\mathbf{R}(\mathbf{a})(\boldsymbol{\Omega})$ & $\boldsymbol{F ( a )}(\mathbf{m})$ \\
\hline 0.5 & 13.81 & 3.63 \\
1 & 6.92 & 6.57 \\
1.5 & 4.62 & 9.62 \\
2 & 3.63 & 12.71 \\
3 & 2.76 & 18.95 \\
4 & 2.32 & 25.21 \\
6 & 1.79 & 37.75 \\
8 & 1.45 & 50.30 \\
10 & 1.22 & 62.86 \\
12 & 1.05 & 75.42 \\
\hline
\end{tabular}

Figure 5 shows the apparent resistivity, $\rho_{a p p}(a)$, of the different models of the geometrical function $F(a)$. The model labeled IEEE1, as seen in case study 1 , is the model that is the closest to both the 'real' model and the data measured, even at small values of $a$. However, the IEEE2 model clearly departed from this behavior, again suggesting the critical character of the L parameter in Equation (1) when electrodes of different length are used. The figure indicates the values measured for this soil using triangular markers. 


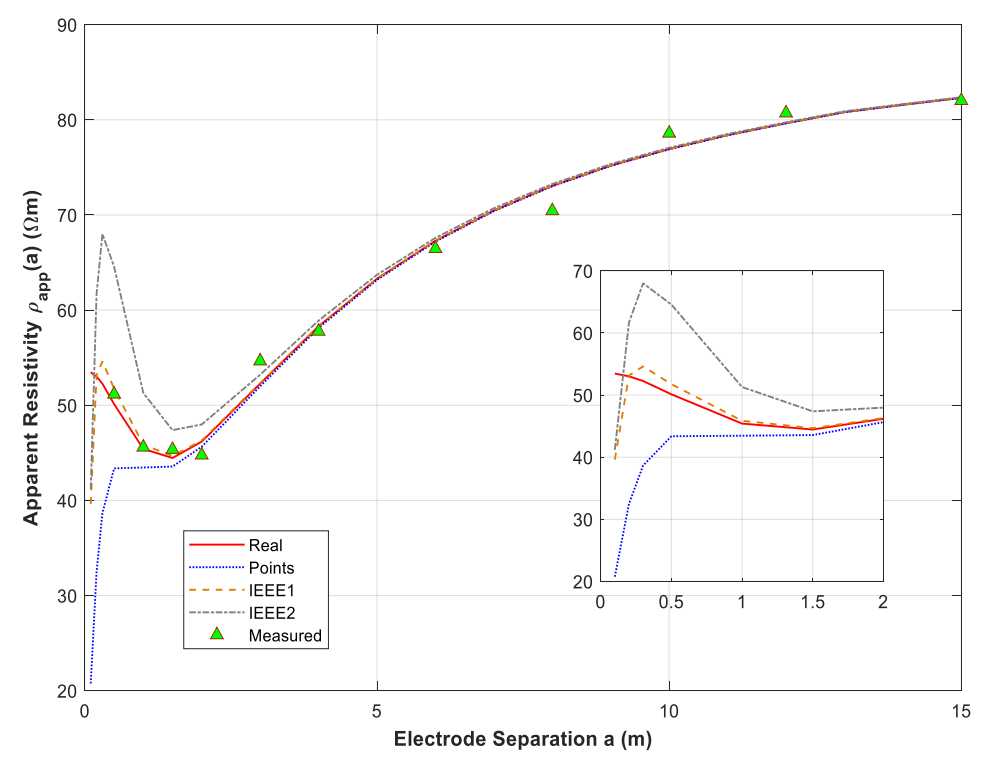

Figure 5. Curves of apparent resistivity vs. the separation $a$ between the electrodes for the measured site in Vitoria (Spain), according to a three-layer soil model. Besides the actual measured data (green triangles), those simulated using the geometrical functions $F(a)$ proposed in the text are shown.

\subsection{Case Study 3}

As a final case study, a new synthetic three-layer model with parameters, $\rho_{1}=10 \Omega \mathrm{m}$, $\rho_{2}=100 \Omega \mathrm{m}, \rho_{3}=50 \Omega \mathrm{m}, h_{1}=2 \mathrm{~m}$, and $h_{2}=3 \mathrm{~m}$, was considered and a VES was simulated using the electrodes in Figure 6. Electrode 1 had the shape of an inverted uppercase $Y$, with branches of the same size and thickness, $\mathrm{L}_{1}=0.3 \mathrm{~m}$, and $\mathrm{r}_{1}=7 \mathrm{~mm}$. Electrode 4 had the shape of an inverted truncated cone of radius $r_{\max }=5 \mathrm{~mm}$ and $r_{\min }=1 \mathrm{~mm}$, and $\mathrm{L}_{4}=0.5 \mathrm{~m}$. The remaining electrodes were analogous to those shown in Figure 2. The set of electrodes was chosen to try to take advantage of a previously buried conductor of known properties, setting it up as electrode 1 . Note that the distance between the electrodes was defined here as the common distance between the upper ends of the electrodes.

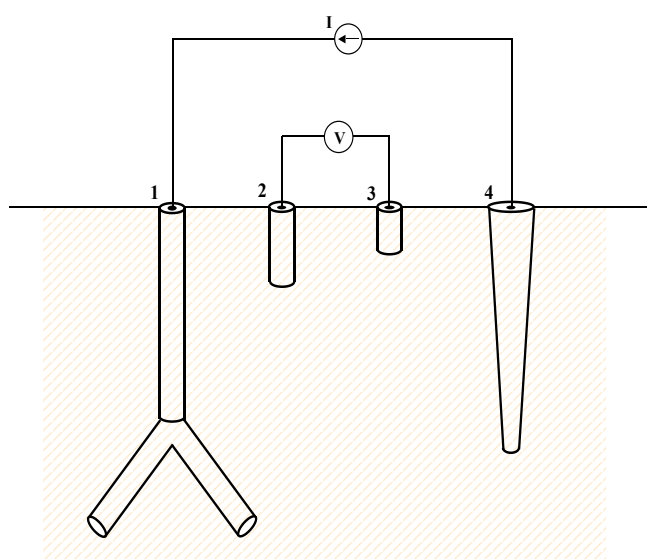

Figure 6. The new set of electrodes for the simulated VES of case study 3.

Figure 7 shows the apparent resistivity, $\rho_{a p p}(a)$, for the different models of $F(a)$ when a VES was performed in the synthetic three-layer soil with previously defined parameters. Again, it can be seen that the 'real' model was the one that best accounted for the values of the apparent resistivity. It was also verified that the IEEE1 model, which entails the definition of $L$ in Equation (1) as the mean value 
of the z-coordinates of the lower ends of the electrodes, was the one that was closest to the curve of the 'real' model.

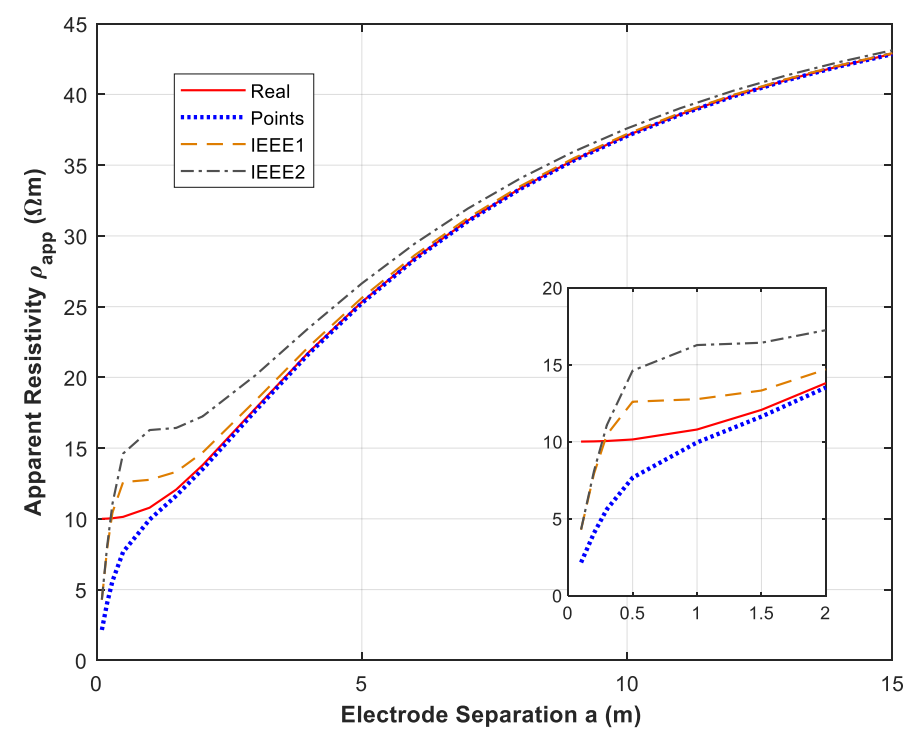

Figure 7. Curves of apparent resistivity vs. the separation, $a$, between the electrodes for the synthetic three-layer soil model for all the considered $F(a)$ models when the set of electrodes from Figure 6 are used.

\section{Conclusions}

On some occasions, a non-standard set of electrodes are used as Wenner probes to measure apparent resistivity. In these cases, the measurement of apparent resistivity at shallow depth may contain significant errors that must be corrected. In this work, it was shown that it is possible to use electrodes of different shape, size, and thickness in a Wenner arrangement, as long as they can be treated as thin wires. Once the resistance measurements have been made, it is necessary to convert them into values of apparent resistivity. This is usually done using expressions such as Equation (2), with $F(a)=2 \pi a$. However, these expressions provide incorrect values for distances between electrodes that can reach up to $5 \mathrm{~m}$. To be precise, the previous model can provide differences in resistivity of $10 \%$ for values of $a<1 \mathrm{~m}$. Although this may seem to be a short distance, note that many grounding system electrodes are buried between 0.5 and $1 \mathrm{~m}$ deep, thus knowing the resistivity at that depth is a very important matter.

The model of Equation (1) can theoretically only be applied to equal electrodes of length, $L$, the parameter contained in Equation (1). When unequal electrodes are used, $L$ does not make sense and must be defined. In this work, two values for $L$ were defined. The so-called IEEE1 model considers $L$ as the mean of the lengths, while for the IEEE2 model, $L$ was the average of the two longest electrodes. In this work it was possible to verify that the IEEE1 model produced better results, although for small values of $a$ it continued to supply incorrect values for the apparent resistivity.

This model, based on the calculation of the geometrical function $F(a)$ from a simple process of calibration of the electrodes, is the only one that supplies the best resistivity values for any value of the separation $a$ between electrodes. Electrodes that differ in size and thickness can be used, with the sole exception that they cannot be too long to avoid crossing the interface between the first layers. Finally, electrodes of different shapes within the thin wire approach can also be used as a probe whenever a parameter equivalent to the usual distance between electrodes, $a$, can be clearly defined.

Author Contributions: All the authors contributed both to the creation of the software and to the execution of the calculations to obtain the results. All the authors discussed both the content and conclusions of the paper.

Funding: This research received no external funding. 
Acknowledgments: The authors would like to thank both the Department of Applied Mathematics and the IEEF Department of the Escuela Técnica Superior de Ingeniería y Diseño Industrial (ETSIDI) at the Polytechnic University of Madrid (UPM) for their support for the undertaking of the research summarized here. We also would like to thank Iberdrola Distribución S.A. for the support provided to perform this work. Furthermore, the authors appreciate the useful suggestions and selfless assistance of Prof. A. Vitores. Finally, the authors thank Ms. Gabriela Andres for her contribution to the linguistic correction of the original paper.

Conflicts of Interest: The authors declare no conflicts of interest.

\section{References}

1. Samouelian, A.; Cousin, I.; Bruand, A.T.A.; Richard, G. Electrical resistivity survey in soil science: A review. Soil Tillage Res. 2005, 83, 173-193. [CrossRef]

2. Van Nostrand, R.G.; Cook, K.L. Interpretation of Resistivity Data; Geological Survey Professional Paper 499; USGS: Reston, VA, USA, 1966.

3. Hesse, A.; Jolivet, A.; Tabbagh, A. New prospects in shallow depth electrical surveying for archaeological and pedological applications. Geophysics 1986, 51, 585-594. [CrossRef]

4. Grard, R. A quadrupolar array for measuring the complex permittivity of the ground: Application to Earth prospection and planetary exploration. Meas. Sci. Technol. 1990, 1, 295-301. [CrossRef]

5. Wenner, F. A Method of Measuring Earth Resistivity; Scientific Paper, Report No. 258; National Bureau of Standards: Gaithersburg, MD, USA, 1916; Volume 12, pp. 469-482.

6. Jacubas, A.; Jablonski, P. The influence of electrode size on resistance measurement in the modified four-electrode method. Measurement 2017, 108, 34-40. [CrossRef]

7. Sunde, E.D. Earth Conduction Effects in Transmission Systems; Dover Publications Inc.: Mineola, NY, USA, 1949.

8. Olowofela, J.A.; Jolaosho, V.O. Measuring the electrical resistivity of the Earth using a fabricated resistivity meter. Eur. J. Phys. 2005, 26, 501. [CrossRef]

9. IEEE Guide for Measuring Earth Resistivity, Ground Impedance, and Earth Surface Potentials of a Grounding System; IEEE Standard 81; IEEE: Piscataway, NJ, USA, 2012.

10. Faleiro, E.; Asensio, G.; Moreno, J. Improved measurements of the apparent resistivity for small depths in Vertical Electrical Soundings. J. Appl. Geophys. 2016, 131, 117-122. [CrossRef]

11. Takahashi, T.; Kawase, T. Analysis of apparent resistivity in a multi-layer earth structur. IEEE Trans. Power Deliv. 1990, 5, 604-612. [CrossRef]

12. Harrington, R.F. Field Computation by Moment Methods; IEEE Press: New York, NY, USA, 1993.

13. Seedher, H.R.; Arora, J.K. Estimation of two layer soil parameters using finite Wenner resistivity expressions. IEEE Trans. Power Deli. 1992, 7, 1213-1217. [CrossRef]

14. Gupta, P.K.; Niwas, S.; Gaurz, V.K. Straightforward inversion of vertical electrical sounding data. Geophysics 1997, 62, 775-785. [CrossRef]

(C) 2019 by the authors. Licensee MDPI, Basel, Switzerland. This article is an open access article distributed under the terms and conditions of the Creative Commons Attribution (CC BY) license (http:/ / creativecommons.org/licenses/by/4.0/). 